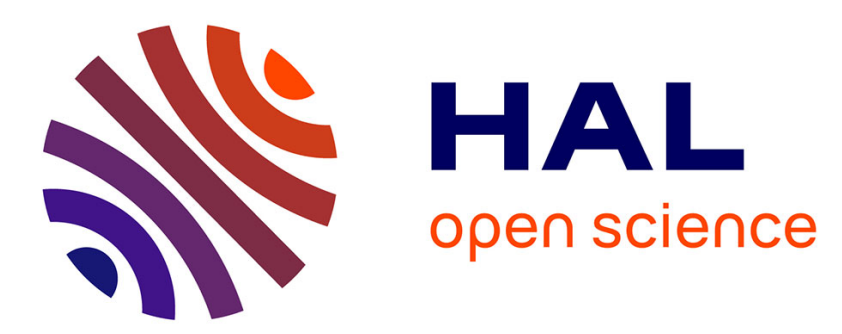

\title{
The inverse problem of determining several coefficients in a nonlinear Lotka-Volterra system
}

Lionel Roques, Michel Cristofol

\section{To cite this version:}

Lionel Roques, Michel Cristofol. The inverse problem of determining several coefficients in a nonlinear Lotka-Volterra system. Inverse Problems, 2012, 28 (7), 16 p. 10.1088/0266-5611/28/7/075007 . hal01264033

\section{HAL Id: hal-01264033 \\ https://hal.science/hal-01264033}

Submitted on 29 Jan 2016

HAL is a multi-disciplinary open access archive for the deposit and dissemination of scientific research documents, whether they are published or not. The documents may come from teaching and research institutions in France or abroad, or from public or private research centers.
L'archive ouverte pluridisciplinaire HAL, est destinée au dépôt et à la diffusion de documents scientifiques de niveau recherche, publiés ou non, émanant des établissements d'enseignement et de recherche français ou étrangers, des laboratoires publics ou privés. 


\title{
The inverse problem of determining several coefficients in a nonlinear Lotka-Volterra system
}

\author{
Lionel Roques $^{1}$ and Michel Cristofol ${ }^{2}$ \\ 1 UR 546 Biostatistique et Processus Spatiaux, INRA, F-84000 Avignon, France \\ 2 Aix-Marseille Université, LATP, Faculté des Sciences et Techniques \\ Avenue Escadrille Normandie-Niemen, F-13397 Marseille Cedex 20, France \\ E-mail: ${ }^{1}$ lionel.roques@avignon.inra.fr, ${ }^{2}$ cristo@latp.univ-mrs.fr
}

\begin{abstract}
In this paper, we prove a uniqueness result in the inverse problem of determining several non-constant coefficients of a system of two parabolic equations which corresponds to a Lotka-Volterra competition model. Our result gives a sufficient condition for the uniqueness of the determination of four coefficients of the system. This sufficient condition only involves pointwise measurements of the solution $(u, v)$ of the system and of the spatial derivative $\partial u / \partial x$ or $\partial v / \partial x$ of one component at a single point $x_{0}$, during a time interval $(0, \varepsilon)$. Our results are illustrated by numerical computations.
\end{abstract}

Keywords: Parabolic equation · Uniqueness · Pointwise measurements · Heterogeneous media $\cdot$ Competition model 


\section{Introduction}

This papers deals with an inverse problem of coefficient determination in a system of two parabolic equations with spatially heterogeneous coefficients. The system that we consider corresponds to a Lotka-Volterra competition model. In this model, the unknowns typically correspond to biological species which are in competition with each other. This type of model is widely used in theoretical as well as more applied works, see e.g. $[1,2,3]$ for Lotka-Volterra competition models based on ordinary differential equations and $[4,5]$ for spatial competition models using systems of partial differential equations of the parabolic type. In these models, the dynamics of the species critically depends on the precise value of the coefficients: depending on these coefficients, the species may coexist or not $[6,4,7]$. The aim of our study is to determine these coefficients using only partial measurements of the species concentrations.

For scalar parabolic equations, uniqueness and stability results in the inverse problem of coefficient determination are generally obtained using the method of Carleman estimates $[8,9]$. This method requires, among other measurements, the knowledge of the solution $u(\theta, x)$ of the equation at some time $\theta>0$ and for all $x$ in the domain $\Omega$ (see $[10,11,12,13,14,15]$ ). Uniqueness and stability results can also be derived from boundary measurements. In particular, there is a huge literature on the determination of nonlinear spatially homogeneous terms $f(u)$ in scalar reaction-diffusion equations from such boundary measurements [16, 17, 18, 19, 20, 21].

In the one-dimensional scalar case, more recent approaches $[22,23]$ lead to uniqueness results for one or several spatially-varying coefficients under the assumption that the solution $u\left(t, x_{0}\right)$ and its spatial derivative $\frac{\partial u}{\partial x}\left(t, x_{0}\right)$ are known at a single point $x_{0}$ for all $t \in(0, \varepsilon)$ and that the initial condition $u(0, x)$ is known over $\Omega$.

Here, we obtain comparable uniqueness results for several coefficients in a system of two nonlinear parabolic equations, using only local or boundary measurements. The previous results on inverse problems of coefficient determination in systems of parabolic equations were based on the method of Carleman estimates [24, 11, 25, 26]. Consequently, as in the scalar case, these methods used measurements of the solution of the model in the whole domain $\Omega$ at some positive time $\theta$. Uniqueness results had not been previously carried out on the basis of local (i.e., without the measurement at $t=\theta$ ) or boundary measurements. Besides, apart from [26] where the authors deal with a system involving a linear equation coupled with a nonlinear equation, the previous works were only concerned with systems of linear equations.

The next section is devoted to the clear formulation of our assumptions and to the statement of our main results. These results are proved in Section 3 and are illustrated by numerical computations in Section 4 . 


\section{Assumptions and main results}

Consider a single-species model:

$$
\frac{\partial u}{\partial t}=D_{1} \frac{\partial^{2} u}{\partial x^{2}}+r_{1}(x) u-a_{11}(x) u^{2}, \text { for } t>0, x \in(a, b) \subset \mathbb{R},
$$

where the diffusion coefficient $D_{1}$ is a positive constant, the intrinsic growth rate $r_{1}$ belongs to $C^{0, \eta}([a, b])$, for some $\eta \in(0,1]$ (The space $C^{0, \eta}$ corresponds to Hölder continuous functions with exponent $\eta$, see e.g. [27]), and the intraspecific competition coefficient $a_{11}$ is positive and also belongs to $C^{0, \eta}([a, b])$.

Assume that a second species enters in competition with species 1 , and that the two-species system can be modelled by the Lotka-Volterra competition model:

$$
\left\{\begin{array}{l}
\frac{\partial u}{\partial t}=D_{1} \frac{\partial^{2} u}{\partial x^{2}}+r_{1} u-a_{11} u^{2}-a_{12} u v, \\
\frac{\partial v}{\partial t}=D_{2} \frac{\partial^{2} v}{\partial x^{2}}+r_{2} v-a_{21} u v-a_{22} v^{2}
\end{array} \text { for } t>0, x \in(a, b) \subset \mathbb{R} .\right.
$$

As in (2.1), $D_{2}>0$ is a constant corresponding to the diffusion coefficient of the second species, $r_{2} \in C^{0, \eta}([a, b])$ is the 2 nd species intrinsic growth rate and $a_{22}>0$ corresponds to the 2 nd species intraspecific competition coefficient $\left(a_{22} \in C^{0, \eta}([a, b])\right)$.

Since the system is competitive, we have:

$$
a_{12}, a_{21}>0 \text { on }[a, b] \text {. }
$$

We furthermore assume that $a_{12}$ is constant and $a_{21} \in C^{0, \eta}([a, b])$. These two coefficients respectively measure the impact of species 2 upon species 1 (resp. of species 1 upon species 2).

Apart from $D_{2}$, we assume that all the coefficients associated with species 2 are unknown: $r_{2}(x), a_{21}(x), a_{22}(x)$ and $a_{12}$ are not known. Our aim is to study under which conditions these coefficients can be uniquely determined by measurements of the solution $(u, v)$.

\section{Initial and boundary conditions}

We assume that $u$ and $v$ satisfy the initial conditions

$$
u(0, x)=u_{0}, v(0, x)=v_{0}
$$

and the boundary conditions:

$$
\left\{\begin{array}{cc}
\alpha_{1} u(t, a)-\beta_{1} \frac{\partial u}{\partial x}(t, a)=0, & \gamma_{1} u(t, b)+\delta_{1} \frac{\partial u}{\partial x}(t, b)=0, \\
\alpha_{2} v(t, a)-\beta_{2} \frac{\partial v}{\partial x}(t, a)=0, & \gamma_{2} v(t, b)+\delta_{2} \frac{\partial v}{\partial x}(t, b)=0
\end{array} \text { for } t>0\right.
$$

with

$$
\alpha_{i}^{2}+\beta_{i}^{2}>0 \text { and } \delta_{i}^{2}+\gamma_{i}^{2}>0, \text { for } i=1,2 \text {. }
$$


These general boundary conditions include the classical Dirichlet case $\left(\beta_{i}=\delta_{i}=0\right.$, for $i=1,2)$ and Neumann case $\left(\alpha_{i}=\gamma_{i}=0\right.$, for $\left.i=1,2\right)$.

In order to deal with classical solutions $(u, v)$ of $(2.2)$, we furthermore make the following hypotheses on the initial conditions:

$$
u_{0}, v_{0} \in C^{2, \eta}([a, b]),
$$

that is $u_{0}$ and $v_{0}$ are $C^{2}$ function such that $\left(u_{0}\right)^{\prime \prime}$ and $\left(v_{0}\right)^{\prime \prime}$ are Hölder continuous. In addition to that, we assume the following compatibility conditions:

$$
\begin{cases}\alpha_{1} u_{0}(a)-\beta_{1}\left(u_{0}\right)^{\prime}(a)=0 & \text { and }\left(u_{0}\right)^{\prime \prime}(a)=0 \text { if } \beta_{1}=0, \\ \gamma_{1} u_{0}(b)+\delta_{1}\left(u_{0}\right)^{\prime}(b)=0 & \text { and }\left(u_{0}\right)^{\prime \prime}(b)=0 \text { if } \delta_{1}=0, \\ \alpha_{2} v_{0}(a)-\beta_{2}\left(v_{0}\right)^{\prime}(a)=0 & \text { and }\left(v_{0}\right)^{\prime \prime}(a)=0 \text { if } \beta_{2}=0 \\ \gamma_{2} v_{0}(b)+\delta_{2}\left(v_{0}\right)^{\prime}(b)=0 & \text { and }\left(v_{0}\right)^{\prime \prime}(b)=0 \text { if } \delta_{2}=0\end{cases}
$$

Under these assumptions, the problem (2.2) admits a unique solution $(u, v)$ with $u, v \in C_{1}^{2}([0, \infty) \times[a, b])$. Existence, uniqueness and regularity of the solution $(u, v)$ are classical (see e.g. [28], Chapter 8).

\section{Hypotheses on the unknown coefficients}

As above-mentioned, the coefficient $a_{12}$ is assumed to be constant. Our assumptions on the coefficients $r_{2}, a_{21}$, and $a_{22}$ is that they belong to the following functional space $\mathcal{M}$ :

$$
\mathcal{M}:=\left\{\psi \in C^{0, \eta}([a, b]) \text { s. t. } \psi \text { is piecewise analytic on }(a, b)\right\} .
$$

A continuous function $\psi$ is called piecewise analytic if there exist $n \geq 1$ and an increasing sequence $\left(\kappa_{j}\right)_{1 \leq j \leq n}$ such that $\kappa_{1}=a, \kappa_{n}=b$, and

$$
\psi(x)=\sum_{j=1}^{n-1} \chi_{\left[\kappa_{j}, \kappa_{j+1}\right)}(x) \varphi_{j}(x),
$$

for all $x \in(a, b)$; here $\varphi_{j}$ are some analytic functions defined on the intervals $\left[\kappa_{j}, \kappa_{j+1}\right]$, and $\chi_{\left[\kappa_{j}, \kappa_{j+1}\right)}$ are the characteristic functions of the intervals $\left[\kappa_{j}, \kappa_{j+1}\right)$ for $j=1, \ldots, n-1$. In particular, if $\psi \in \mathcal{M}$, then, for each $x \in[a, b)$ (resp. $x \in(a, b])$, there exists $r=r_{x}>0$ such that $\psi$ is analytic on $[x, x+r]$ (resp. $[x-r, x])$.

Note that the assumption $\psi \in \mathcal{M}$ is not very restrictive. For instance, the set of piecewise linear functions on $[a, b]$ is a subset of $\mathcal{M}$.

\section{Measurements of the solution}

Our results use three measurements of the solution of the system (2.2), starting from three different couples of initial conditions. More precisely, We consider three couples of initial conditions $\left(u_{0}^{1}, v_{0}^{1}\right),\left(u_{0}^{2}, v_{0}^{2}\right),\left(u_{0}^{3}, v_{0}^{3}\right)$ such that

$$
u_{0}^{1}, u_{0}^{2}, u_{0}^{3}, v_{0}^{1}, v_{0}^{2}, v_{0}^{3}>0 \text { in }(a, b),
$$


and

$$
\left(u_{0}^{3}-u_{0}^{2}\right)\left(v_{0}^{2}-v_{0}^{1}\right)-\left(v_{0}^{3}-v_{0}^{2}\right)\left(u_{0}^{2}-u_{0}^{1}\right) \neq 0 \text { in }(a, b) .
$$

In other terms, for all $x \in(a, b)$, the points $\left(u_{0}^{1}, v_{0}^{1}\right)(x),\left(u_{0}^{2}, v_{0}^{2}\right)(x)$ and $\left(u_{0}^{3}, v_{0}^{3}\right)(x)$ belong to the positive quadrant and are misaligned.

We assume that for some $x_{0} \in(a, b)$, the solution $(u, v)$ of $(2.2)$ and its spatial derivative can be measured at the position $x_{0}$. More precisely, for some $\varepsilon>0$ and for each couple of initial conditions $\left(u_{0}^{k}, v_{0}^{k}\right)$, with $k=1,2,3$, we assume that $(u, v)\left(t, x_{0}\right)$ and $\frac{\partial u}{\partial x}\left(t, x_{0}\right)$ (or $\frac{\partial v}{\partial x}\left(t, x_{0}\right)$, see Theorem 2.1 below) can be measured for all $t \in(0, \varepsilon)$.

In the next section, Theorem 2.1 shows that these measurements are sufficient to determine the four unknown coefficients $r_{2}(x), a_{21}(x), a_{22}(x)$ and $a_{12}$, and consequently the solution $(u, v)$ of $(2.2)$ on $(0, \infty) \times(a, b)$.

Main result

Let $\tilde{a}_{12} \in(0, \infty)$ and $\tilde{r}_{2}, \tilde{a}_{21}, \tilde{a}_{22}$ belong to $\mathcal{M}$. Let $(\tilde{u}, \tilde{v})$ be the solution of:

$$
\left\{\begin{array}{l}
\frac{\partial \tilde{u}}{\partial t}=D_{1} \frac{\partial^{2} \tilde{u}}{\partial x^{2}}+r_{1} \tilde{u}-a_{11} \tilde{u}^{2}-\tilde{a}_{12} \tilde{u} \tilde{v}, \\
\frac{\partial \tilde{v}}{\partial t}=D_{2} \frac{\partial^{2} \tilde{v}}{\partial x^{2}}+\tilde{r}_{2} \tilde{v}-\tilde{a}_{21} \tilde{u} \tilde{v}-\tilde{a}_{22} \tilde{v}^{2}
\end{array} \text { for } t>0, x \in(a, b) \subset \mathbb{R},\right.
$$

with the initial conditions:

$$
\tilde{u}(0, x)=u_{0}, \tilde{v}(0, x)=v_{0} .
$$

Theorem 2.1. Let $x_{0} \in(a, b)$ and $\varepsilon>0$. Assume that for each couple of initial conditions $\left(u_{0}^{k}, v_{0}^{k}\right)$, with $k=1,2,3$, and for all $t \in(0, \varepsilon)$, we have:

$$
\left\{\begin{aligned}
(u, v)\left(t, x_{0}\right) & =(\tilde{u}, \tilde{v})\left(t, x_{0}\right), \\
\frac{\partial u}{\partial x}\left(t, x_{0}\right) & =\frac{\partial \tilde{u}}{\partial x}\left(t, x_{0}\right) \text { or } \frac{\partial v}{\partial x}\left(t, x_{0}\right)=\frac{\partial \tilde{v}}{\partial x}\left(t, x_{0}\right) .
\end{aligned}\right.
$$

Then, $\tilde{a}_{12}=a_{12}$ and $\tilde{r}_{2} \equiv r_{2}, \tilde{a}_{21} \equiv a_{21}, \tilde{a}_{22} \equiv a_{22}$ in $(a, b)$.

If the second derivative $\frac{\partial^{2} \tilde{u}}{\partial x^{2}}\left(t, x_{0}\right)$ is known, the coefficients $r_{2}(x), a_{21}(x), a_{22}(x)$ and $a_{12}$ can be determined without any measurement of the function $v$. This is the consequence of our next result.

Corollary 2.2. Let $x_{0} \in(a, b)$ and $\varepsilon>0$. Assume that for each couple of initial conditions $\left(u_{0}^{k}, v_{0}^{k}\right)$, with $k=1,2,3$, we have:

$$
\left\{\begin{aligned}
u\left(t, x_{0}\right) & =\tilde{u}\left(t, x_{0}\right), \\
\frac{\partial u}{\partial x}\left(t, x_{0}\right) & =\frac{\partial \tilde{u}}{\partial x}\left(t, x_{0}\right), \quad \text { for all } t \in(0, \varepsilon) . \\
\frac{\partial^{2} u}{\partial x^{2}}\left(t, x_{0}\right) & =\frac{\partial^{2} \tilde{u}}{\partial x^{2}}\left(t, x_{0}\right),
\end{aligned}\right.
$$

Then, $\tilde{a}_{12}=a_{12}$ and $\tilde{r}_{2} \equiv r_{2}, \tilde{a}_{21} \equiv a_{21}, \tilde{a}_{22} \equiv a_{22}$ in $(a, b)$. 
Remarks 2.3. $\quad$ - The conclusions of Theorem 2.1 and Corollary 2.2 are still valid when $x_{0}=a$ and $\beta_{1}, \beta_{2} \neq 0$ (resp. $x_{0}=b$ and $\delta_{1}, \delta_{2} \neq 0$ ) if the initial conditions $u_{0}^{i}, v_{0}^{i}$ are assumed to be positive in $[a, b)$ (resp. $\left.(a, b]\right)$.

- The result of Corollary 2.2 implies that, for any subset $\omega \subset(a, b)$, all the unknown coefficients $r_{2}, a_{22}, a_{12}, a_{21}$ are uniquely determined on $[a, b]$ by a measurement of $u$ on $\omega$ during a time period $(0, \varepsilon)$.

A counter-example

The results of Theorem 2.1 and Corollary 2.2 are not true in general if the initial conditions $\left(u_{0}^{k}, v_{0}^{k}\right)$ do not verify the assumption (2.10). We construct here a counterexample to these results in a case where the assumption (2.10) is not verified. This counter-example also shows that our results are not true in general if the number of initial conditions is less than three.

Assume that the boundary conditions are of the Neumann type $\left(\alpha_{i}=\gamma_{i}=0\right.$, for $i=1,2)$ and that all the coefficients $r_{1}, r_{2}, a_{11}, a_{12}, a_{21}$ and $a_{22}$ are constant. Assume furthermore that there exists $\lambda>0$ such that $r_{2}=\lambda r_{1}, a_{21}=\lambda a_{11}$ and $a_{22}=\lambda a_{12}$. Now, take three couples $\left(u_{0}^{k}, v_{0}^{k}\right), k=1,2,3$, of constant and positive initial conditions on the straight line $\left\{(x, y), \mid a_{11} x+a_{12} y=r_{1}\right\}$. Then, each couple $\left(u_{0}^{k}, v_{0}^{k}\right)$ is a solution of:

$$
\left\{\begin{array}{l}
r_{1} u_{0}^{k}-a_{11}\left(u_{0}^{k}\right)^{2}-a_{12} u_{0}^{k} v_{0}^{k}=0, \\
r_{2} v_{0}^{k}-a_{21} u_{0}^{k} v_{0}^{k}-a_{22}\left(v_{0}^{k}\right)^{2}=0,
\end{array}\right.
$$

for $k=1,2,3$. Thus, each couple $\left(u_{0}^{k}, v_{0}^{k}\right)$ is also a stationary solution of $(2.2)$. Note that the couples $\left(u_{0}^{k}, v_{0}^{k}\right), k=1,2,3$, are aligned and therefore do not verify the assumption (2.10).

Let $\tilde{\lambda}>0$ verify $\tilde{\lambda} \neq \lambda$, and take $\tilde{r}_{2}=\tilde{\lambda} r_{1}, \tilde{a}_{21}=\tilde{\lambda} a_{11}$ and $\tilde{a}_{22}=\tilde{\lambda} a_{12}$. Again, the three couples $\left(u_{0}^{k}, v_{0}^{k}\right)$ verify the system:

$$
\left\{\begin{array}{l}
r_{1} u_{0}^{k}-a_{11}\left(u_{0}^{k}\right)^{2}-a_{12} u_{0}^{k} v_{0}^{k}=0 \\
\tilde{r}_{2} v_{0}^{k}-\tilde{a}_{21} u_{0}^{k} v_{0}^{k}-\tilde{a}_{22}\left(v_{0}^{k}\right)^{2}=0
\end{array}\right.
$$

and are therefore stationary solutions of (2.11). In particular, the assumptions (2.12) and (2.13) of Theorem 2.1 and Corollary 2.2 are fulfilled at any point $x_{0} \in(a, b)$ and for all $\varepsilon>0$. However, the conclusions of Theorem 2.1 and Corollary 2.2 are not true, since $\tilde{r}_{2} \neq r_{2}, \tilde{a}_{21} \neq a_{21}$ and $\tilde{a}_{22} \neq a_{22}$.

\section{Proof of Theorem 2.1 and Corollary 2.2}

Proof of Theorem 2.1

We set $U=u-\tilde{u}, V=\tilde{v}-v, R=r_{2}-\tilde{r}_{2}$ and $A_{i j}=a_{i j}-\tilde{a}_{i j}$ for all $i, j \in\{1,2\}$ (and $(i, j) \neq(1,1))$. 
Remark 3.1. It would be more natural to set $U=u-\tilde{u}$ and $V=v-\tilde{v}$, as it is done in most papers involving inverse problems of coefficient determination in systems [24, 11, 25, 26]. It is noteworthy that here we have set $U=u-\tilde{u}$ and $V=\tilde{v}-v$. This enables us to obtain a "cooperative" system for $(U, V)$. In such cooperative systems, an increase of $U$ has a positive effect on $V$ and similarly, an increase of $V$ has a positive effect on U. Such systems are known to satisfy a comparison principle (see Chap. 3, Sec. 8 in [29]). Note that, when the number of species is 3 or more, it is not possible in general to convert a competitive system into a cooperative system.

Whatever the initial condition $\left(u_{0}^{k}, v_{0}^{k}\right)$, with $k=1 \ldots 3$, the couple $(U, V)$ verifies:

$$
\left\{\begin{array}{l}
\frac{\partial U}{\partial t}-D_{1} \frac{\partial^{2} U}{\partial x^{2}}=U\left[r_{1}-a_{11}(u+\tilde{u})-\tilde{a}_{12} v\right]+\tilde{a}_{12} \tilde{u} V-A_{12} u v \\
\frac{\partial V}{\partial t}-D_{2} \frac{\partial^{2} V}{\partial x^{2}}=V\left[\tilde{r}_{2}-\tilde{a}_{22}(v+\tilde{v})-\tilde{a}_{21} u\right]+\tilde{a}_{21} \tilde{v} U-v\left[R-A_{21} u-A_{22} v\right]
\end{array}\right.
$$

and $U(0, x)=V(0, x)=0$ for all $x \in(a, b)$. Evaluating the first equation in (3.14) at $(t, x)=\left(0, x_{0}\right)$ and using assumption $(2.12)$ we obtain:

$$
A_{12} u_{0}\left(x_{0}\right) v_{0}\left(x_{0}\right)=0 .
$$

Thus, using e.g. the initial condition $\left(u_{0}, v_{0}\right)=\left(u_{0}^{1}, v_{0}^{1}\right)$, we get $A_{12}=0$. Thus, $\tilde{a}_{12}=a_{12}$ and system (3.14) can be rewritten:

$$
\left\{\begin{array}{l}
\frac{\partial U}{\partial t}-D_{1} \frac{\partial^{2} U}{\partial x^{2}}=U\left[r_{1}-a_{11}(u+\tilde{u})-a_{12} v\right]+a_{12} \tilde{u} V \\
\frac{\partial V}{\partial t}-D_{2} \frac{\partial^{2} V}{\partial x^{2}}=V\left[\tilde{r}_{2}-\tilde{a}_{22}(v+\tilde{v})-\tilde{a}_{21} u\right]+\tilde{a}_{21} \tilde{v} U-v\left[R-A_{21} u-A_{22} v\right]
\end{array}\right.
$$

Let us set:

$$
\mathcal{A}_{+}=\left\{x \geq x_{0} \text { s.t. } R(y) \equiv A_{21}(y) \equiv A_{22}(y) \equiv 0 \text { for all } y \in\left[x_{0}, x\right]\right\},
$$

and

$$
x_{1}:= \begin{cases}\sup \left(\mathcal{A}_{+}\right) & \text {if } \mathcal{A}_{+} \text {is not empty } \\ x_{0} & \text { if } \mathcal{A}_{+} \text {is empty. }\end{cases}
$$

If $x_{1}=b$, then $R(y) \equiv A_{21}(y) \equiv A_{22}(y) \equiv 0$ on $\left[x_{0}, b\right]$. Let us assume on the contrary that $x_{1}<b$.

Step 1. Assume that $x_{1}<b$. We prove that there exists an initial condition $\left(u_{0}^{k^{*}}, v_{0}^{k^{*}}\right)$, with $k^{*} \in\{1,2,3\}$ such that for some $\theta \in(0, T)$, and $x_{2} \in\left(x_{1}, b\right)$ we have $R(x)-A_{21}(x) u(t, x)-A_{22}(x) v(t, x) \neq 0$ for all $(t, x) \in[0, \theta] \times\left(x_{1}, x_{2}\right]$.

By assumption, $x_{1}<b$. Thus, from the definition of the set $\mathcal{M}$, there exists $\delta>0$ such that $x_{1}+\delta<b$ and $R, A_{21}, A_{22}$ are analytic on $\left[x_{1}, x_{1}+\delta\right]$ and not all identically 
zero. Thus, the integer

$$
\rho=\max \left\{\rho ^ { \prime } \in \mathbb { N } \text { such that } \left\{\begin{array}{rl}
R(x) & =O\left(\left(x-x_{1}\right)^{\rho^{\prime}}\right), \\
\left.A_{21}(x)=O\left(\left(x-x_{1}\right)^{\rho^{\prime}}\right), \quad \text { as } x \rightarrow x_{1}^{+}\right\} . \\
A_{22}(x)=O\left(\left(x-x_{1}\right)^{\rho^{\prime}}\right),
\end{array}\right.\right.
$$

is well-defined.

Furthermore, the function $(t, x) \mapsto R(x)-A_{21}(x) u(t, x)-A_{22}(x) v(t, x)$ can then be written as

$$
(t, x) \mapsto\left(x-x_{1}\right)^{\rho} \times\left[\hat{R}(x)-\hat{A}_{21}(x) u(t, x)-\hat{A}_{22}(x) v(t, x)\right],
$$

for all $(t, x) \in \mathbb{R}_{+} \times\left[x_{1}, x_{1}+\delta\right]$, where the functions $\hat{R}, \hat{A}_{21}$ and $\hat{A}_{22}$ are analytic and not all zero at the point $x_{1}$. From our assumption (2.10) on the initial conditions, there necessarily exists $k^{*} \in\{1,2,3\}$ such that $\hat{R}\left(x_{1}\right)-\hat{A}_{21}\left(x_{1}\right) u_{0}^{k^{*}}\left(x_{1}\right)-\hat{A}_{22}\left(x_{1}\right) v_{0}^{k^{*}}\left(x_{1}\right) \neq 0$.

By continuity of the solution $(u, v)$ of $(2.2)$ with initial condition $\left(u_{0}^{k^{*}}, v_{0}^{k^{*}}\right)$, the application $(t, x) \mapsto \hat{R}(x)-\hat{A}_{21}(x) u(t, x)-\hat{A}_{22}(x) v(t, x)$ is continuous in $[0, \infty) \times$ $\left[x_{1}, x_{1}+\delta\right)$. In particular, there exist $\theta>0$ and $x_{2} \in\left(x_{1}, x_{1}+\delta\right)$ such that $\hat{R}(x)-\hat{A}_{21}(x) u(t, x)-\hat{A}_{22}(x) v(t, x) \neq 0$ for all $(t, x)$ in $[0, \theta] \times\left[x_{1}, x_{2}\right]$. Using (3.17) we obtain

$$
R(x)-A_{21}(x) u(t, x)-A_{22}(x) v(t, x) \neq 0,
$$

for all $(t, x) \in[0, \theta] \times\left(x_{1}, x_{2}\right]$.

Step 2. We show that if $\left(u_{0}, v_{0}\right)=\left(u_{0}^{k^{*}}, v_{0}^{k^{*}}\right)$, there exists $\varepsilon^{\prime}>0$ such that either $U>0$ and $V>0$ or $U<0$ and $V<0$ in $\left(0, \varepsilon^{\prime}\right) \times\left(x_{0}, x_{2}\right)$.

From the previous step, we know that if the initial condition $\left(u_{0}, v_{0}\right)$ is set to $\left(u_{0}^{k^{*}}, v_{0}^{k^{*}}\right)$, the application $(t, x) \mapsto R(x)-A_{21}(x) u(t, x)-A_{22}(x) v(t, x)$ has a constant strict sign in $[0, \theta] \times\left(x_{1}, x_{2}\right]$.

Case (a) Assume that $R(x)-A_{21}(x) u(t, x)-A_{22}(x) v(t, x)<0$ in $[0, \theta] \times\left(x_{1}, x_{2}\right]$. Since $U(0, x)=0$ and $V(0, x)=0$ for all $x \in(a, b)$, it follows from (3.16) that

$$
\frac{\partial V}{\partial t}\left(0, x_{2}\right)=-v\left[R-A_{21} u-A_{22} v\right]\left(0, x_{2}\right)>0 .
$$

Next, we show that

$$
\frac{\partial U}{\partial t}\left(0, x_{2}\right)=0 \text { and } \frac{\partial^{2} U}{\partial t^{2}}\left(0, x_{2}\right)>0 .
$$

Indeed, evaluating the first equation in (3.16) at $t=0$, we easily get the equality $\frac{\partial U}{\partial t}(0, x)=0$ for all $x \in(a, b)$. The proof of the inequality in $(3.20)$ is more involved. 
Let $W$ be the (classical) solution of the equation:

$$
\left\{\begin{array}{l}
\frac{\partial W}{\partial t}=D_{1} \frac{\partial^{2} W}{\partial x^{2}}+\frac{\partial}{\partial t}\left(U\left[r_{1}-a_{11}(u+\tilde{u})-a_{12} v\right]\right)+\frac{\partial}{\partial t}\left(a_{12} \tilde{u}\right) V+a_{12} \tilde{u} \frac{\partial V}{\partial t}, \\
\alpha_{1} W(t, a)-\beta_{1} \frac{\partial W}{\partial x}(t, a)=0, \gamma_{1} W(t, b)+\delta_{1} \frac{\partial W}{\partial x}(t, b)=0, \\
W(0, x)=0
\end{array}\right.
$$

for all $t>0$ and $x \in(a, b)$.

Now, let us set $\hat{W}=\frac{\partial U}{\partial t}$. Differentiating the equation satisfied by $U$ in (3.16) with respect to $t$, and using Theorems 4 and 5 in [30] Chap. 7 (which readily extend to more general boundary conditions such as those in (3.21)), we obtain that $\hat{W}$ is the unique weak solution of (3.21). It follows that $\hat{W}=W$ and therefore $\hat{W}$ is a classical solution of (3.21). As a consequence, $U \in C_{2}^{2}([0, \infty) \times(a, b))$ and $\frac{\partial^{2} U}{\partial t^{2}}(t, x)=\frac{\partial W}{\partial t}(t, x)$ for all $(t, x) \in[0, \infty) \times(a, b)$. Computing the equation (3.21) at $t=0$ and $x=x_{2}$, and using the equalities $U\left(0, x_{2}\right)=\frac{\partial U}{\partial t}\left(0, x_{2}\right)=V\left(0, x_{2}\right)=0$, we get:

$$
\frac{\partial^{2} U}{\partial t^{2}}\left(0, x_{2}\right)=\frac{\partial W}{\partial t}\left(0, x_{2}\right)=a_{12} u_{0}^{k^{*}}\left(x_{2}\right) \frac{\partial V}{\partial t}\left(0, x_{2}\right)>0
$$

from assumptions (2.3), (2.9) and from (3.19). This proves (3.20).

From (3.19) and (3.20), and since $U\left(0, x_{2}\right)=V\left(0, x_{2}\right)=0$, we know that there exists $\varepsilon^{\prime} \in(0, \varepsilon)$ such that $U\left(t, x_{2}\right)>0$ and $V\left(t, x_{2}\right)>0$ in $\left(0, \varepsilon^{\prime}\right)$. Besides, from the assumption (2.12) of Theorem 2.1, we know that $U\left(t, x_{0}\right)=V\left(t, x_{0}\right)=0$ for all $t \in\left(0, \varepsilon^{\prime}\right)$. As a consequence, the couple $(U, V)$ verifies:

$$
\left\{\begin{array}{l}
\left\{\begin{array}{l}
\frac{\partial U}{\partial t}-D_{1} \frac{\partial^{2} U}{\partial x^{2}}-U\left[r_{1}-a_{11}(u+\tilde{u})-a_{12} v\right]=a_{12} \tilde{u} V, \\
\frac{\partial V}{\partial t}-D_{2} \frac{\partial^{2} V}{\partial x^{2}}-V\left[\tilde{r}_{2}-\tilde{a}_{22}(v+\tilde{v})-\tilde{a}_{21} u\right]>\tilde{a}_{21} \tilde{v} U,
\end{array} \quad t \in\left(0, \varepsilon^{\prime}\right), x \in\left(x_{0}, x_{2}\right),\right. \\
U\left(t, x_{0}\right)=0, U\left(t, x_{2}\right)>0, t \in\left(0, \varepsilon^{\prime}\right), \\
V\left(t, x_{0}\right)=0, V\left(t, x_{2}\right)>0, t \in\left(0, \varepsilon^{\prime}\right), \\
U(0, x)=V(0, x)=0, x \in\left(x_{0}, x_{2}\right) .
\end{array}\right.
$$

By continuity of $(\tilde{u}, \tilde{v})$ with respect to $t$ and from assumption (2.9) on the initial conditions, we known that for $t>0$ small enough $\tilde{u}(t, x)>0$ and $\tilde{v}(t, x)>0$ for all $x \in\left(x_{0}, x_{2}\right)$. Thus, even if it means decreasing $\varepsilon^{\prime}$, we can assume that $\tilde{u}(t, x)>0$ and $\tilde{v}(t, x)>0$ in $\left(0, \varepsilon^{\prime}\right) \times\left(x_{0}, x_{2}\right)$. As a consequence, and since $a_{12}$ and $\tilde{a}_{21}$ are assumed to be positive, the system (3.23) satisfies a monotonicity assumption:

$$
\frac{\partial}{\partial V}\left(a_{12} \tilde{u} V\right)=a_{12} \tilde{u}>0 \text { and } \frac{\partial}{\partial U}\left(\tilde{a}_{21} \tilde{v} U\right)=\tilde{a}_{21} \tilde{v}>0 \text { in }\left(0, \varepsilon^{\prime}\right) \times\left(x_{0}, x_{2}\right) .
$$

Finally, the couple $(U, V)$ satisfies all the assumptions of the strong maximum principle for systems satisfying the monotonicity assumption (3.24) (Theorem 13 p. 190 in [29]), which implies that either $(U, V)>0$ in $\left(0, \varepsilon^{\prime}\right) \times\left(x_{0}, x_{2}\right)$ or there exists some time $t_{0} \in\left(0, \varepsilon^{\prime}\right)$ such that $U \equiv 0$ or $V \equiv 0$ in $\left(0, t_{0}\right) \times\left(x_{0}, x_{2}\right)$. From the boundary condition satisfied by $U$ and $V$ at $x_{2}$, we necessarily have $(U, V)>0$ in $\left(0, \varepsilon^{\prime}\right) \times\left(x_{0}, x_{2}\right)$. 
Case (b) Assume that $R(x)-A_{21}(x) u(t, x)-A_{22}(x) v(t, x)>0$ in $[0, \theta] \times\left(x_{1}, x_{2}\right]$. Applying the same arguments as above to $-U$ and $-V$, we obtain that $U<0$ and $V<0$ in $\left(0, \varepsilon^{\prime}\right) \times\left(x_{0}, x_{2}\right)$.

Step 3. Using Hopf's Lemma and assumption (2.12), we get a contradiction with the assumption of Step 1.

From Step 2, we know that either $U>0$ and $V>0$ or $U<0$ and $V<0$ in $\left(0, \varepsilon^{\prime}\right) \times\left(x_{0}, x_{2}\right]$. Assume that $U>0$ and $V>0$ in $\left(0, \varepsilon^{\prime}\right) \times\left(x_{0}, x_{2}\right)$ (the case $U<0$ and $V<0$ can be treated the same way). Then,

$$
\frac{\partial U}{\partial t}-D_{1} \frac{\partial^{2} U}{\partial x^{2}}-U\left[r_{1}-a_{11}(u+\tilde{u})-a_{12} v\right]>0 \text { in }\left(0, \varepsilon^{\prime}\right) \times\left(x_{0}, x_{2}\right),
$$

and

$$
\frac{\partial V}{\partial t}-D_{2} \frac{\partial^{2} V}{\partial x^{2}}-V\left[\tilde{r}_{2}-\tilde{a}_{22}(v+\tilde{v})-\tilde{a}_{21} u\right]>0 \text { in }\left(0, \varepsilon^{\prime}\right) \times\left(x_{0}, x_{2}\right) .
$$

Since $U>0$ and $V>0$ in $\left(0, \varepsilon^{\prime}\right) \times\left(x_{0}, x_{2}\right)$ and $U\left(t, x_{0}\right)=V\left(t, x_{0}\right)=0$, the Hopf's Lemma (Theorem 14 p. 190 in [29]) implies that

$$
\frac{\partial U}{\partial x}\left(t, x_{0}\right)>0 \text { and } \frac{\partial V}{\partial x}\left(t, x_{0}\right)>0 \text { for all } t \in\left(0, \varepsilon^{\prime}\right),
$$

which contradicts the assumption (2.12) of Theorem 2.1. As a consequence, the assumption $x_{1}<b$ of Step 1 is false.

\section{Step 4. Conclusion.}

From Step 3, we know that $x_{1}=b$. Therefore $R(y) \equiv A_{21} \equiv A_{22}(y) \equiv 0$ on $\left[x_{0}, b\right]$. Setting:

$$
\mathcal{A}_{-}=\left\{x \leq x_{0} \text { s.t. } R(y) \equiv A_{21}(y) \equiv A_{22}(y) \equiv 0 \text { for all } y \in\left[x, x_{0}\right]\right\}
$$

and

$$
y_{1}:= \begin{cases}\inf \left(\mathcal{A}_{-}\right) & \text {if } \mathcal{A}_{-} \text {is not empty } \\ x_{0} & \text { if } \mathcal{A}_{-} \text {is empty }\end{cases}
$$

we can prove, by applying the same arguments as above, that $y_{1}=a$ and consequently $R(y) \equiv A_{21}(y) \equiv A_{22}(y) \equiv 0$ on $\left[a, x_{0}\right]$.

Finally, $R(y) \equiv A_{21}(y) \equiv A_{22}(y) \equiv 0$ on $[a, b]$ which concludes the proof of Theorem 2.1 . 
Proof of Corollary 2.2

Assume that $u$ verifies (2.13). Take any initial data $\left(u_{0}^{k}, v_{0}^{k}\right)$. As already observed, evaluating the first equation in (3.14) at $(t, x)=\left(0, x_{0}\right)$ and using the assumption (2.13) we obtain that $\tilde{a}_{12}=a_{12}$. Rewriting the equations satisfied by $u$ and $\tilde{u}$, we get:

$$
\left\{\begin{array}{l}
\frac{\partial u}{\partial t}=D_{1} \frac{\partial^{2} u}{\partial x^{2}}+r_{1} u-a_{11} u^{2}-a_{12} u v, \\
\frac{\partial \tilde{u}}{\partial t}=D_{1} \frac{\partial^{2} \tilde{u}}{\partial x^{2}}+r_{1} \tilde{u}-a_{11} \tilde{u}^{2}-a_{12} \tilde{u} \tilde{v}
\end{array} \text { for } t>0, x \in(a, b) \subset \mathbb{R} .\right.
$$

Using assumption (2.13), we obtain:

$$
\left\{\begin{array}{l}
\frac{\partial u}{\partial t}=D_{1} \frac{\partial^{2} u}{\partial x^{2}}+r_{1} u-a_{11} u^{2}-a_{12} u v, \\
\frac{\partial u}{\partial t}=D_{1} \frac{\partial^{2} u}{\partial x^{2}}+r_{1} u-a_{11} u^{2}-a_{12} u \tilde{v}
\end{array} \text { for } t \in(0, \varepsilon), x=x_{0} .\right.
$$

From assumption (2.9), and by continuity of $u$ with respect to $t$, we know that $u\left(t, x_{0}\right)>0$ for $t$ small enough and for any initial data $\left(u_{0}^{k}, v_{0}^{k}\right)$. Finally, it follows from (3.28) that

$$
v\left(t, x_{0}\right)=\tilde{v}\left(t, x_{0}\right) \text { for all } t \in\left(0, \varepsilon^{\prime}\right),
$$

for some $\varepsilon^{\prime} \in(0, \varepsilon)$. The result of Corollary then follows from Theorem 2.1.

\section{Numerical computations}

In this section, we check whether the measurements (2.12) of Theorem 2.1 allow for an accurate reconstruction of the coefficients $r_{2}, a_{21}, a_{22}$ and $a_{12}$.

Given the initial data $\left(u_{0}^{k}, v_{0}^{k}\right)$, for $k=1,2,3$, and the measurements $\left(u^{k}, v^{k}\right)\left(t, x_{0}\right)$ and $\partial u^{k} / \partial x\left(t, x_{0}\right)$ of the corresponding solution of $(2.2)$ for $t \in(0, \varepsilon)$, we can look for the vector of coefficients $\Gamma=\left(a_{12}, r_{2}, a_{21}, a_{22}\right)$ as a minimizer of some functional $G_{\Gamma}$. Indeed, define the set $\mathcal{M}^{+}$as the subset of $\mathcal{M}$ made of positive functions. Then, for any $\tilde{\Gamma}=\left(\tilde{a}_{12}, \tilde{r}_{2}, \tilde{a}_{21}, \tilde{a}_{22}\right) \in(0, \infty) \times \mathcal{M} \times \mathcal{M}^{+} \times \mathcal{M}^{+}$, the distance between our measurements and the solutions $\left(\tilde{u}^{k}, \tilde{v}^{k}\right)$ associated with the vector of coefficients $\tilde{\Gamma}$ can be evaluated through the function:

$$
G_{\Gamma}(\tilde{\Gamma})=\sum_{k=1}^{3}\left\|\left(u^{k}, v^{k}\right)\left(\cdot, x_{0}\right)-\left(\tilde{u}^{k}, \tilde{v}^{k}\right)\left(\cdot, x_{0}\right)\right\|_{\left(L^{2}(0, \varepsilon)\right)^{2}}+\left\|\frac{\partial u^{k}}{\partial x}\left(\cdot, x_{0}\right)-\frac{\partial \tilde{u}^{k}}{\partial x}\left(\cdot, x_{0}\right)\right\|_{L^{2}(0, \varepsilon)} .
$$

Then, $G_{\Gamma}(\Gamma)=0$ and from Theorem 2.1 this is the unique global minimum of $G_{\Gamma}$ in $(0, \infty) \times \mathcal{M} \times \mathcal{M}^{+} \times \mathcal{M}^{+}$.

In our numerical computations, we fixed $(a, b)=(0,1), D_{1}=0.1, D_{2}=0.2$, $r_{1}=a_{11}=1$ and $\alpha_{i}=\gamma_{i}=0$, for $i=1,2$ (Neumann boundary conditions). The solution was measured at the point $x_{0}=2 / 3$ and during the time-interval $(0, \varepsilon)=(0,0.3)$.

Besides, we assumed that the vector of coefficients $\Gamma$ belongs to a finite-dimensional subspace of $(0, \infty) \times \mathcal{M} \times \mathcal{M}^{+} \times \mathcal{M}^{+}$. Let $E$ be the $n+1-\operatorname{dimensional~}(n=9$ in our 
computations) subspace of $\mathcal{M}$ defined by:

$$
\begin{aligned}
E:=\left\{\rho \in C^{0, \eta}([0,1]) \mid \exists\left(h_{j}\right)_{0 \leq j \leq n}\right. & \in \mathbb{R}^{n+1}, \\
\rho(x) & \left.=\sum_{j=0}^{n} h_{j} \cdot J\left((n-2)\left(x-c_{j}\right)\right) \text { on }[0,1]\right\},
\end{aligned}
$$

with $c_{j}=\frac{j-1}{n-2}$ and $J(x)=\frac{(x-2)^{4}(x+2)^{4}}{2^{8}}$ if $x \in(-2,2)$, and $J(x)=0$ otherwise. Let $E^{+}$be the set of positive functions in $E$. We assumed that $\Gamma \in(0, \infty) \times E \times E^{+} \times E^{+}$.

We randomly drawn 30 vectors with a uniform law in $(0,10) \times(-5,5)^{10} \times(0,10)^{10} \times$ $(0,10)^{10}$. Each vector corresponds to an element $\Gamma_{i}$ of $(0, \infty) \times E \times E^{+} \times E^{+}$. Starting from the constant initial data $\left(u_{0}^{1}, v_{0}^{1}\right)=(0.1,0.5),\left(u_{0}^{2}, v_{0}^{2}\right)=(0.5,0.2)$ and $\left(u_{0}^{3}, v_{0}^{3}\right)=$ $(0.9,0.5)$, the corresponding values of $\left(u^{k}, v^{k}\right)\left(t, x_{0}\right)$ and $\partial u^{k} / \partial x\left(t, x_{0}\right)$ for $k=1,2,3$, were recorded $\ddagger$. This enabled us to compute $G_{\Gamma_{i}}(\tilde{\Gamma})$ for any $\tilde{\Gamma}$ in $(0, \infty) \times E \times E^{+} \times E^{+}$. The minimizations $\oint$ of the functions $G_{\Gamma_{i}}$ led to 30 vectors of coefficients $\Gamma_{i}^{*}$, each one corresponding to a computed minimizer of the function $G_{\Gamma_{i}}$.

The average values of the quantities $\left\|r_{2}-r_{2}^{*}\right\|_{L^{2}(0,1)},\left\|a_{21}-a_{21}^{*}\right\|_{L^{2}(0,1)},\left\|a_{22}-a_{22}^{*}\right\|_{L^{2}(0,1)}$ and $\left|a_{12}-a_{12}^{*}\right|$ over the 30 samples of vectors $\Gamma_{i}$ were $4 \cdot 10^{-2}, 5 \cdot 10^{-2}, 6 \cdot 10^{-2}$ and $5 \cdot 10^{-6}$, respectively. These values correspond to a very accurate estimation of $\Gamma$. This is illustrated by Fig. 1, which depicts an example of vector of coefficients $\Gamma$ in $(0, \infty) \times E \times E^{+} \times E^{+}$, together with the vector of coefficients $\Gamma^{*}$ which was obtained by minimizing $G_{\Gamma}$.

$\ddagger$ The numerical computations of $(u, v)$ and $(\tilde{u}, \tilde{v})$ were carried out with Comsol Multiphysics ${ }^{\circledR}$ timedependent solver. We used a second order finite element method (FEM) with 960 elements. This solver uses a method of lines approach incorporating variable order and variable stepsize backward differentiation formulas.

$\S$ The minimization of the functions $G_{\Gamma}$ was performed using MATLAB's ${ }^{\circledR}$ fminunc solver. This optimization algorithm uses a Quasi-Newton method with a mixed quadratic and cubic line search procedure. The stopping criterion was $G_{\Gamma_{i}}<10^{-6}$. 


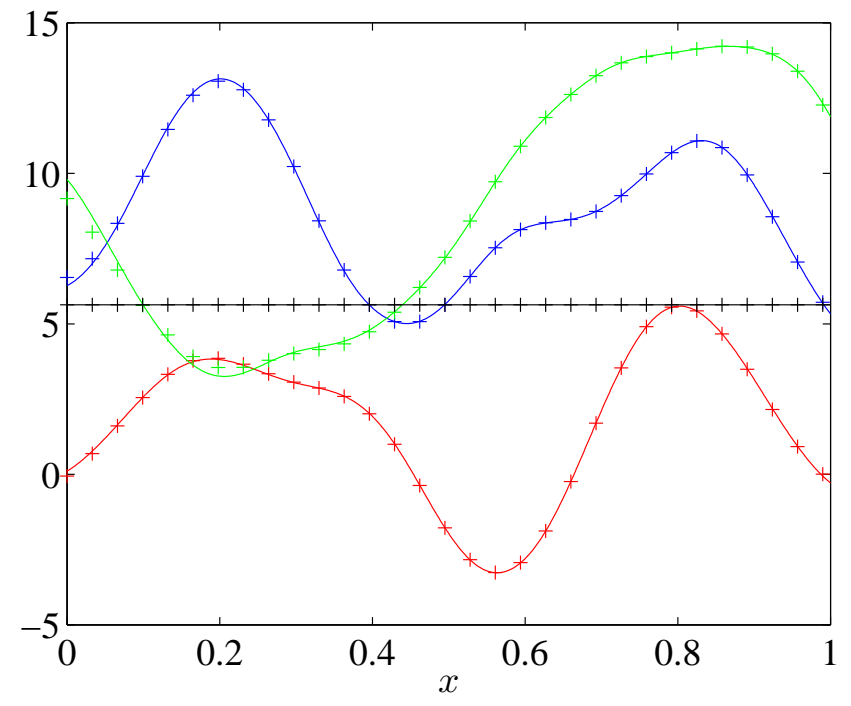

Figure 1. An example of vector of coefficients $\Gamma$ together with the vector of coefficients $\Gamma^{*}$ obtained by minimizing $G_{\Gamma}$. Plain lines: the coefficients $r_{2}$ (in red), $a_{21}$ (in blue) and $a_{22}$ (in green). The black line corresponds to a constant value $a_{12}$. Crosses: the functions $r_{1}^{*}$ (in red), $a_{21}^{*}$ (in blue), $a_{22}^{*}$ (in green) and $a_{12}^{*}$ obtained by minimizing $G_{\Gamma=\left(a_{12}, r_{2}, a_{21}, a_{22}\right)}$. In this particular example, we have $\left\|r_{2}-r_{2}^{*}\right\|_{L^{2}(0,1)}=$ $3 \cdot 10^{-2},\left\|a_{21}-a_{21}^{*}\right\|_{L^{2}(0,1)}=5 \cdot 10^{-2},\left\|a_{22}-a_{22}^{*}\right\|_{L^{2}(0,1)}=0.1$, and $\left|a_{12}-a_{12}\right|=5 \cdot 10^{-7}$. 


\section{References}

[1] R MacArthur and R Levins. The limiting similarity, convergence, and divergence of coexisting species. Am Nat, 101(921):377-385, 1967.

[2] Robert M May and W J Leonard. Nonlinear aspects of competition between three species. SIAM Journal on Applied Mathematics, 29(2):243, 1975.

[3] S Smale. On the differential equations of species in competition. Journal of Mathematical Biology, 3(1):5-7, 1976.

[4] S Cantrell, R and C Cosner. Spatial ecology via reaction-diffusion equations. John Wiley \& Sons Ltd, Chichester, UK , 2003.

[5] Richard Durrett and Simon Levin. The importance of being discrete (and spatial). Theor Popul Biol, 46(3):363-394, 1994.

[6] Robert Stephen Cantrell and Chris Cosner. On the effects of spatial heterogeneity on the persistence of interacting species. Journal of Mathematical Biology, 37(2):103-145, 1998.

[7] L Roques and M D Chekroun. Probing chaos and biodiversity in a simple competition model. Ecological Complexity, 8:98-104, 2011.

[8] A L Bukhgeim and M V Klibanov. Uniqueness in the large of a class of multidimensional inverse problems. Soviet Mathematics - Doklady, 24:244-247, 1981.

[9] M V Klibanov and A Timonov. Carleman estimates for coefficient inverse problems and numerical applications. Inverse And Ill-Posed Series, VSP, Utrecht, 2004.

[10] M Belassoued and M Yamamoto. Inverse source problem for a transmission problem for a parabolic equation. Journal of Inverse and Ill-Posed Problems, 14(1):47-56, 2006.

[11] A Benabdallah, M Cristofol, P Gaitan, and M Yamamoto. Inverse problem for a parabolic system with two components by measurements of one component. Applicable Analysis, 88(5):683-710, 2009.

[12] M Choulli. Une introduction aux problmes inverses elliptiques et paraboliques. Springer, 2009.

[13] M Cristofol and L Roques. Biological invasions: Deriving the regions at risk from partial measurements. Math Biosci, 215(2):158-166, 2008.

[14] O Y Immanuvilov and M Yamamoto. Lipschitz stability in inverse parabolic problems by the Carleman estimate. Inverse Problems, 14:1229-1245, 1998.

[15] M Yamamoto and J Zou. Simultaneous reconstruction of the initial temperature and heat radiative coefficient. Inverse Problems, 17:1181-1202, 2001.

[16] M Choulli, E M Ouhabaz, and M Yamamoto. Stable determination of a semilinear term in a parabolic equation. Communications on Pure and Applied Analysis, 5:447-462, 2006.

[17] P DuChateau and W Rundell. Unicity in an inverse problem for an unknown reaction term in a reaction-diffusion equation. Journal of Differential Equations, 59:155-164, 1985.

[18] H Egger, H W Engl, and M Klibanov. Global uniqueness and Hölder stability for recovering a nonlinear source term in a parabolic equation. Inverse Problems, 21:271-290, 2005.

[19] A Lorenzi. An inverse problem for a semilinear parabolic equation. Annali di Matematica Pura ed Applicata, 131:145-166, 1982.

[20] S Nakamura. A note on uniqueness in an inverse problem for a semilinear parabolic equation. Nihonkai Mathematical Journal, 12:71-73, 2001.

[21] M S Pilant and W Rundell. An inverse problem for a nonlinear parabolic equation. Communications in Partial Differential Equations, 11:445-457, 1986.

[22] L Roques and M Cristofol. On the determination of the nonlinearity from localized measurements in a reaction-diffusion equation. Nonlinearity, 23:675-686, 2010.

[23] M Cristofol, J Garnier, F Hamel, and L Roques. Uniqueness from pointwise observations in a multi-parameter inverse problem. Communications on Pure and Applied Mathematics, in press, 2011.

[24] A Benabdallah, M Cristofol, P Gaitan, and L De Teresa. A new carleman inequality for parabolic systems with a single observation and applications. Comptes Rendus Mathematique, 348(1- 
2):25-29, 2010.

[25] M Cristofol, P Gaitan, and H Ramoul. Inverse problems for a two by two reaction-diffusion system using a carleman estimate with one observation. Inverse Problems, 22:1561-1573, 2006.

[26] M Cristofol, P Gaitan, H Ramoul, and M Yamamoto. Identification of two independant coefficients with one observation for a nonlinear parabolic system. Applicable Analysis, in press.

[27] A Friedman. Partial differential equations of parabolic type. Prentice-Hall, Englewood Cliffs, NJ, 1964.

[28] C V Pao. Nonlinear Parabolic and Elliptic Equations. Plenum Press, New York, 1992.

[29] M H Protter and H F Weinberger. Maximum Principles in Differential Equations. Prentice-Hall, Englewood Cliffs, NJ, 1967.

[30] L C Evans. Partial differential equations. University of California, Berkeley - AMS, 1998. 